\title{
Osteogenic Differentiation of Human Mesenchymal Stem Cells in Freeze-Gelled Chitosan/Nano $\beta$-Tricalcium Phosphate Porous Scaffolds Crosslinked with Genipin
}

\author{
Nadeem Siddiqui, ${ }^{1}$ Krishna Pramanik, ${ }^{1}$ Esmaiel Jabbari ${ }^{2}$ \\ ${ }^{1}$ Department of Biotechnology and Medical Engineering, National Institute of Technology, \\ Rourkela, 769008, India. \\ ${ }^{2}$ Biomimetic Materials and Tissue Engineering Laboratory, Department of Chemical \\ Engineering, \\ University of South Carolina, Columbia, South Carolina, USA
}

Submitted to

\section{Corresponding author:}

Esmaiel Jabbari, Ph.D.

Associate Professor of Chemical and Biomedical Engineering

Swearingen Engineering Center, Rm 2C11

University of South Carolina

Columbia, SC 29208

Tel: $\quad$ (803) 777-8022

Fax: (803) 777-0973

Email: jabbari@mailbox.sc.edu 


\begin{abstract}
The objective of this work was to investigate material properties and osteogenic differentiation of human mesenchymal stem cells (hMSCs) in genipin (GN) crosslinked chitosan/nano $\beta$-tricalcium phosphate (CS/nano $\beta$-TCP) scaffolds, and compare the results with tripolyphosphate (TPP) crosslinked scaffolds. Porous crosslinked CS/nano $\beta$-TCP scaffolds were produced by freeze-gelation using GN (CBG scaffold) and TPP (CBT scaffold) as crosslinkers. The prepared CBT and CBG scaffolds were characterized with respect to porosity, pore size, water content, wettability, compressive strength, mass loss, and osteogenic differentiation of hMSCs. All scaffolds displayed interconnected honeycomb-like microstructures. There was a significant difference between the average pore size, porosity, contact angle, and percent swelling of CBT and CBG scaffolds. The average pore size of CBG scaffolds was higher than CBT, the porosity of $\mathrm{CBG}$ was lower than $\mathrm{CBT}$, the water contact angle of $\mathrm{CBG}$ was higher than CBT, and the percent swelling of CBG was lower than CBT. At a given crosslinker concentration, there was not a significant difference in compressive modulus and mass loss of CBG and CBT scaffolds. Metabolic activity of hMSCs seeded in CBG scaffolds was slightly higher than CBT. Furthermore, CBG scaffolds displayed slightly higher extent of mineralization after 21 days incubation in osteogenic medium compared to CBT.
\end{abstract}

Key Words: chitosan; freeze gelation; genipin crosslinking; microstructure; human MSCs; osteogenesis 


\section{Introduction}

Bone tissue defects and diseases are increasing worldwide due to the shortage of organ donor. Allograft bone is used extensively in regeneration of large defects but its use is limited by immune rejection and the risk of disease transfer $[1,2]$. To overcome these issues, tissue engineering has emerged as an alternative strategy to repair and replace diseased and/or damaged bone tissue through the development of biological substitutes that enable complete recovery of the tissue function [3]. Bone is considered as the second most transplanted tissue and there is a great demand for bone grafts and substitutes $[4,5]$. The engineered scaffold should be biocompatible and have the desired degradation, mechanical strength, and porosity [6]. Further, the scaffold should stimulate adhesion and osteogenic differentiation of seeded progenitor cells [7].

An attractive approach to bone regeneration is to use hybrid matrices produced by reinforcing natural polymers with ceramic fillers $[8,9]$. Chitosan is a biocompatible and biodegradable natural polymer that has antibacterial activity and stimulates wound healing with free amine groups in its molecular structure for crosslinking [10]. However, chitosan has low mechanical strength and high degradation rate which limits its application as a matrix for delivery of cells in bone regeneration [11]. To overcome these limitations, reinforcement with ceramic fillers and crosslinking has been used to improve strength and decrease degradation [1214]. In our previous work, we developed freeze-gelled CS/nano $\beta$-TCP composite scaffolds with improved mechanical, bioactivity and cell supportive properties $[15,16]$. The present work focuses on osteogenic differentiation of human mesenchymal stem cell (MSCs) in crosslinked CS/nano $\beta$-TCP composite scaffolds. Glutaraldehyde is widely used as a crosslinking agent to reduce deformation of biopolymers but the by-products of its degradation are shown to be 
harmful to cells [17]. As an alternative, the inorganic tripolyphosphate (TPP) has been used to crosslink chitosan and reduce its degradation rate without harmful side effects [18, 19]. Genipin (GN) a natural compound extracted from Gardenia fruits has been used as a crosslinking agent for synthesis of elastic gels in wound healing [20,21]. The objective of this work was to compare the effect of GN with TPP crosslinking for CS/nano $\beta$-TCP composite scaffolds produced by freeze-gelation on viability and osteogenic differentiation of human MSCs. The porous scaffolds were evaluated with respect to porosity, wettability, swelling, compressive strength, degradation, and osteogenic differentiation of hMSCs.

\section{Materials and method}

\subsection{Materials}

Chitosan (CS, degree of deacetylation > 85\%), nano $\beta$-TCP $\left[\mathrm{Ca}_{3}\left(\mathrm{PO}_{4}\right)_{2}\right.$ with $>98 \% \beta$ phase and $<200 \mathrm{~nm}$ average size], TPP and GN were purchased from Sigma-Aldrich (St. Louis, MO, USA). Sodium hydroxide pellets, acetic acid, and ethanol were purchased from Merck (Darmstadt, Germany). Other solvents were obtained from VWR (Bristol, CT) and used as received. Dulbecco's phosphate-buffer saline (PBS), Dulbecco's Modified Eagle's Medium (DMEM; $4.5 \mathrm{~g} / \mathrm{L}$ glucose with L-glutamine and without sodium pyruvate), and MTT assay [3(4,5-dimethylthiazol-2-yl)-2,5-diphenyltetrazolium bromide] were purchased from Life Technologies (Grand Island, NY). Fetal bovine serum (FBS) was purchased from Atlas Biologicals (Fort Collins, CO). TRIzol for isolation of cellular RNA and trypsin were purchased from Invitrogen (Carlsbad, CA). Penicillin (PN), streptomycin (SP), fungizone (FG), gentamicin sulfate (GS), dexamethasone (DEX), ascorbic acid (AA), and ß-sodium glycerophosphate ( $\beta \mathrm{GP}$ ) were purchased from Sigma-Aldrich. Quant-it PicoGreen dsDNA reagent kit was obtained from Invitrogen (Carlsbad, CA). QuantiChrom calcium and alkaline phosphatase (ALP) assay kits 
were purchased from Bioassay Systems (Hayward, CA). Human mesenchymal stem cells (hMSCs), harvested and cultured from healthy human bone marrow, were purchased from Lonza (Allendale, NJ). The cells were tested for purity by high expression of CD105, CD166, CD29, and CD44 and low expression of CD14, CD34 and CD45 markers as described by the supplier (Lonza).

\subsection{Methods}

\subsubsection{Development of crosslinked chitosan/nano $\beta$-TCP scaffolds by freeze-gelation}

Chitosan was dissolved in aqueous acetic acid (0.1M) solution to form a $2.5 \mathrm{wt} \%$ CS solution. Nano $\beta$-TCP powder $(0.4 \mathrm{wt} \%)$ was dispersed in deionised (DI) water for $1 \mathrm{~h}$ by ultrasonication and the dispersion was added to CS solution dropwise and stirred overnight to form a homogenous suspension [1]. Different concentrations of GN and TPP $(0.01-0.20 \mathrm{w} / \mathrm{v})$ were added to the chitosan/nano $\beta$-TCP suspension under stirring for $2 \mathrm{~h}$ [12]. The chitosan/nano $\beta$ $\mathrm{TCP} /$ crosslinker suspension was poured into a petri dish, maintained at $-20^{\circ} \mathrm{C}$ for $6 \mathrm{~h}$, and the

frozen suspension was allowed to gel by immersion in a pre-cooled medium for $6 \mathrm{~h}$. The gelation medium composed of 70:30 v/v NaOH:ethanol [15]. The crosslinked sample was removed from the gelation medium, washed with PBS, dried under vacuum for $6 \mathrm{~h}$ at $40^{\circ} \mathrm{C}$, and stored at $-20^{\circ} \mathrm{C}$.

\subsubsection{Morphological analysis and scaffold porosity}

The morphology and pore size of the scaffolds were examined by scanning electron microscopy (SEM JEOL-JSM6480LV, Japan). The scaffold was sputter-coated with platinum (Quorumtech, Q150RES, Czech Republic) prior to imaging. The pore size of the scaffold was determined from SEM images with Image $\mathbf{J}$ software (National Institutes of Health, Bethesda, MD) using minimum of 25 pores. The porosity of the scaffold was measured with a Poremaster-33 mercury 
porosimeter (Quantachrome Instruments, Boynton Beach, FL, USA). The bulk density (BD) of the sample was measured using the formula $\mathrm{BD}=\mathrm{D} /(\mathrm{W}-\mathrm{S})$ where $\mathrm{D}, \mathrm{S}$, and $\mathrm{W}$ are the dry weight, suspended weight, and soaked weight, respectively. The percent volume porosity was calculated from the raw data using the Washburn equation [22].

\subsubsection{X-ray diffraction, FT-IR analysis and contact angle measurement}

XRD was used to characterize crystallinity of the scaffolds. XRD spectrum of the sample was recorded using X'Pert Pro x-ray diffractometer (PANalytical, Westborough, MA, USA) with $\mathrm{CuK} \alpha$ radiation source at $45 \mathrm{kV}$. The data was collected in a $2 \theta$ range of $10-80^{\circ}$ with a scanning rate of $2 \%$ min. FTIR spectrum of the scaffold was collected with an AIM-8800 automated infrared microscope (Shimadzu, Tokyo, Japan). The sample was pelletized using a hydraulic press by mixing with dry potassium bromide $(\mathrm{KBr})$ powder. The instrument was operated in transmittance mode and spectrum was collected in the wavelength region of 500 to $4000 \mathrm{~cm}^{-1}$ with a resolution of $8 \mathrm{~cm}^{-1}$ [23]. The contact angle of the scaffold was measured by a K100MK3 tensiometer (Kruss GmbH, Hamburg, Germany). A droplet of distilled water was applied to the surface of the sample with a detection speed of $6 \mathrm{~mm} / \mathrm{min}$ and sensitivity of $0.001 \mathrm{mN} / \mathrm{m}$ at $25^{\circ} \mathrm{C}$ and $65 \%$ humidity. The mean value of the contact angle was calculated from five individual measurements [3].

\subsubsection{Measurement of compressive strength, swelling, and degradation}

The compressive strength of the scaffolds (10 mm diameter $\mathrm{x} 7 \mathrm{~mm}$ thickness) was measured with a Tinius Olsen testing system (Model H10K-S, Horsham, PA, USA) with a 1000 N load cell and $1 \mathrm{~mm} / \mathrm{min}$ crosshead speed under ambient conditions. Compressive strength (S) of the scaffold was calculated using the formula $S=F_{\max } / A$ where $F_{\max }$ was the maximum applied load and A was the initial cross-sectional area of the scaffold [1]. The extent of swelling of the 
scaffolds was measured in DI water at ambient conditions for up to $24 \mathrm{~h}$ [24]. At each time point, the weight of the sample was measured before (dry weight) and after (swollen weight) swelling. The percent swelling of the scaffold was measured by dividing the difference in weight before and after swelling by the dried weight. The degradation of the scaffolds was measured in a simulated body fluid (SBF) containing lysozyme as described [25]. The initial dry weight $\left(\mathrm{W}_{0}\right)$ of the scaffold was recorded. At each time point, the scaffold was lyophilized and the final dry weight $\left(\mathrm{W}_{\mathrm{t}}\right)$ was recorded. The percent remaining mass was calculated using the formula $100-$ $\left[\left(\mathrm{W}_{0}-\mathrm{W}_{\mathrm{t}}\right) / \mathrm{W}_{0} \times 100\right]$

\subsubsection{Cell seeding and culture}

Prior to cell seeding, the scaffolds were sterilized by treating with $1 \% \mathrm{v} / \mathrm{v}$ solution of antibiotic and antimycotic agents $(10,000$ units/mL penicillin $\mathrm{G}, 10 \mathrm{mg} / \mathrm{mL}$ streptomycin sulphate and 25 $\mu \mathrm{g} / \mathrm{mL}$ amphotericin B) in PBS for $24 \mathrm{~h}$ followed by exposure to ultraviolet radiation under a laminar hood for $6 \mathrm{~h}$ [26]. hMSCs were cultivated at 5000 cells $/ \mathrm{cm}^{2}$ seeding density in a high glucose DMEM medium supplemented with 10\% FBS, 100 units $/ \mathrm{mL}$ penicillin and $100 \mu \mathrm{g} / \mathrm{mL}$ streptomycin. The medium was refreshed every 3 days. After reaching $70 \%$ confluency, the hMSCs were detached with $0.1 \%$ trypsin- $0.03 \%$ EDTA and sub-cultivated in a 1:3 ratio. All hMSCs used in cell culture experiments were passaged <5 times according to supplier's instructions. The scaffolds $\left(1 \mathrm{~cm}^{2}\right.$ by $500 \mu \mathrm{m}$ thickness $)$ were seeded with $5 \times 10^{6}$ cells diluted in PBS and incubated in basal medium for $24 \mathrm{~h}$ to allow for cell attachment as described [27]. Next, the medium was replaced with osteogenic medium (basal medium supplemented with 50 $\mu \mathrm{g} / \mathrm{mL}$ ascorbic acid and $10 \mathrm{mM} \beta$-glycerophosphate, and $100 \mathrm{nM}$ dexamethasone) and the cellseeded scaffold was incubated for 21 days for assessment of osteogenic differentiation and mineralization [27]. 


\subsubsection{Determination of Cell morphology}

The cell seeded scaffold was fixed with $4 \%$ formaldehyde in PBS on ice for $10 \mathrm{~min}$. After washing, the scaffold was stained with $1 \%$ osmium tetroxide solution, washed with PBS, and dehydrated in sequential ethanol solutions (50, 70, 80, 95 and 100\%) as previously described [28]. After air drying, the sample was sputter coated with platinum and imaged with an SEM as described in the previous section.

\subsubsection{Measurement of cell metabolic activity and viability}

Metabolic activity of hMSCs seeded in the scaffold was measured by MTT assay as previously described [29]. At each time point after washing with PBS, the sample was incubated with 0.5 $\mathrm{mg} / \mathrm{mL}$ MTT solution at $37^{\circ} \mathrm{C}$ for $2 \mathrm{~h}$. The MTT assay is based on the conversion of MTT into insoluble formazan crystals by oxidoreductase enzymes in the mitochondria of the cell. After incubation, the medium was replaced with $500 \mu \mathrm{L}$ of DMSO for dissolution of the formazan crystals. Next, the sample was incubated for $4 \mathrm{~h}$ in dark and the absorbance was measured at the peak wavelength of $595 \mathrm{~nm}$ with a Victor X3 Multi-label plate reader (Model 2030, Perklin Elmer, Waltham, MA, USA). For imaging the viable cells, cells were fixed with formaldehyde, permeabilized with $0.1 \%$ Triton X-100 in PBS, and incubated in a solution containing $2 \mu \mathrm{M}$ calcein AM and $2 \mu \mathrm{M}$ ethidium homodimer in HEPES buffer for $30 \mathrm{~min}$. The stained scaffold

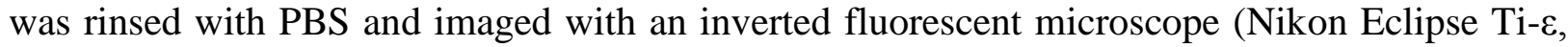
Nikon, Melville, NY) as previously described [28].

\subsubsection{Cell distribution and cytoskeletal organization}

Cell distribution and cytoskeletal organisation of the cells seeded in the scaffolds were analysed by confocal laser scanning microscopy using Hoescht and Alexa Fluor 488 Phalloidin stain according to a previously established protocol [16]. Briefly, the cell-seeded scaffold was fixed 
with $4 \%$ paraformaldehyde for 1 min followed by staining with the Hoescht nuclear stain for 1 min. Next, the scaffold was stained with Phalloidin and examined with a confocal laser scanning microscope (CLSM Leica SP8 inverted microscope, Germany equipped with HeNe $633 \mathrm{~nm}$ laser) at emission and excitation wavelength of $488 \mathrm{~nm}$ and $633 \mathrm{~nm}$, respectively.

\subsubsection{Determination of cellularity, ALP activity, and calcium content}

The hMSC-seeded scaffold was washed with serum-free DMEM for $8 \mathrm{~h}$ to remove serum components, washed with PBS, and lysed with $0.4 \mathrm{~mL}$ lysis buffer (10 $\mathrm{mM}$ tris and $2 \%$ triton) for $1 \mathrm{~h}$. The scaffold with lysed cells was homogenized, centrifuged, and the supernatant was used for determination of cellularity, ALP activity and calcium content. The double stranded DNA (dsDNA) content of the sample was measured using a Quant-it PicoGreen assay according to manufacturer's instructions [14]. An aliquot $(100 \mu \mathrm{L})$ of the working solution was added to $100 \mu \mathrm{L}$ of the cell lysate and incubated for $4 \mathrm{~min}$ at ambient conditions. The fluorescence of the solution was measured with a Synergy HT plate reader at emission and excitation wavelength of 485 and $528 \mathrm{~nm}$, respectively. The measured fluorescent intensity was correlated to cell number using a calibration curve constructed with MSC cells of known concentration ranging from zero to $4 \times 10^{4}$ cells/mL. ALP activity was measured using a QuantiChrom ALP Assay Kit according to manufacturer's instructions [30]. A $10 \mu \mathrm{L}$ aliquot of the sonicated cell lysate was added to $190 \mu \mathrm{L}$ of the reagent solution containing $10 \mathrm{mM}$-nitrophenyl phosphate and $5 \mathrm{mM}$ magnesium acetate and absorbance was recorded at time zero and again after 4 min. ALP

activity was calculated using the equation $\left[\left(A_{t=4}-A_{t=0}\right) /\left(A_{\text {calibrator }}-A_{d d H_{2} O}\right) \times 808\right]$. The absorbance was measured on a Synergy HT plate reader at the wavelength of $405 \mathrm{~nm}$ and normalized to DNA content. Calcium content was measured using a QuantiChrom Calcium Assay Kit according to manufacturer's instructions [30]. A $0.2 \mathrm{~mL}$ of $2 \mathrm{M} \mathrm{HCl}$ was added to 0.2 
$\mathrm{mL}$ aliquot of the sonicated cell lysate to dissolve the calcium content of the mineralized matrix. Next, a $5 \mu \mathrm{L}$ aliquot of the supernatant was added to $200 \mu \mathrm{L}$ of the working solution. After incubation for $3 \mathrm{~min}$, the absorbance was measured on a Synergy HT plate reader at the wavelength of $612 \mathrm{~nm}$. The measured intensity was correlated to equivalent amounts of $\mathrm{Ca}^{2+}$ using a calibration curve constructed with calcium chloride solutions of known concentration ranging from zero to $200 \mu \mathrm{g} / \mathrm{mL}$.

\subsubsection{Statistical analysis}

Data are expressed as means \pm standard deviation. Significant differences between the groups were evaluated using a two-tailed student t-test. A value of $\mathrm{p}<0.05$ was considered as statistically significant.

\section{Results and discussion}

\subsection{Scaffold characterization}

Experimental groups included genipin crosslinked chitosan/ $\beta$-TCP composite scaffold (denoted by $\mathrm{CS} / \beta-\mathrm{TCP} / \mathrm{GN}$ or CBG), and TPP crosslinked chitosan/ $\beta$-TCP composite scaffolds (denoted by $\mathrm{CS} / \beta$-TCP/TPP or CBT). Chitosan and chitosan/ $\beta$-TCP scaffolds without GN or TPP crosslinking are denoted by CS and CS/ $\beta$-TCP, respectively. The concentration of GN and TPP was varied between 0.01 and $2.0 \mathrm{w} / \mathrm{v}$. The concentration of crosslinker in CBG and CBT scaffolds was $0.1 \mathrm{w} / \mathrm{v}$ unless otherwise specified. CBTn and CBGn were used for TPP and GN crosslinked samples, respectively, with " $n$ " w/v crosslinker concentration. For example, the chitosan scaffold reinforced with $\beta$-TCP and crosslinked with $0.1 \mathrm{w} / \mathrm{v}$ genipin was abbreviated by CBG0.1.

\subsection{Morphological analysis and scaffold porosity}


Scaffold porosity and pore size play an important role in the transport of nutrients and oxygen to the cells seeded in the pore volume of scaffold [5]. SEM images (a) through (c) show the porous structure of CBG scaffolds crosslinked with $0.01,0.1$, and $0.2 \mathrm{w} / \mathrm{v}$ GN, respectively, and images (d) through (f) show the structure of CBT scaffolds with TPP concentrations given above. All scaffolds showed highly interconnected honeycomb-like pore structures with thin walls $<10 \mu \mathrm{m}$ in thickness. The average pore size and porosity of CBG and CBT scaffolds as a function of crosslinker concentration is compared with those of CS and CS/ $\beta$-TCP in Table 1 . The average pore size increased with crosslinker concentration for GN and TPP crosslinked scaffolds but CBG scaffolds ( $171 \pm 8$ to $199 \pm 9 \mu \mathrm{m})$ had a significantly higher pore size compared to CBT scaffolds ( $168 \pm 4$ to $186 \pm 5 \mu \mathrm{m}$ ). For a given crosslinker type, there was a significant difference in scaffold pore size and porosity for crosslinker concentrations of 0.01 and $0.2 \mathrm{w} / \mathrm{v}$. As the crosslinker concentration increased from $0.01 \mathrm{w} / \mathrm{v}$ to $0.05,0.10,0.15$ and $0.20 \mathrm{w} / \mathrm{v}$, the porosity of CBT scaffolds decreased from $78 \pm 3 \%$ to $76 \pm 1,75 \pm 2.2,73 \pm 2$ and $71 \pm 2 \%$, respectively, whereas the porosity of CBG scaffolds decreased from $76 \pm 3 \%$ to $74 \pm 2,73 \pm 2,70 \pm 2$ and $68 \pm 2 \%$. The scaffolds formed larger pore sizes as the rigidity of the pores increased with crosslinker concentration. Overall, there was a significant difference in pore size and porosity of CBT and CBG scaffolds. Further, the pore wall thickness increased with GN [Figure 1 (a-c)] and TPP [Figure 1 (d-f)] crosslinking as shown in the SEM images (Figure 1). The results were in good agreement with the previously reported pore size and porosity of chitosan scaffolds [20, 21].

\subsection{XRD, FT-IR and contact angle measurement}

XRD spectra of chitosan (CS), CBG, and CBT scaffolds with $0.1 \mathrm{w} / \mathrm{v}$ concentration of GN or TPP crosslinker are shown in Figure 2a. The broad, low-intensity absorption with peak position of $23^{\circ}$ in the CS spectrum indicated that the chitosan matrix was amorphous. The sharp 
diffraction peaks in the spectra of $\mathrm{CBG}$ and CBT at $2 \Theta=25.8^{\circ}, 31.8^{\circ}, 32^{\circ}$, and $33^{\circ}$ confirmed the presence of $\beta$-TCP in the scaffold [31]. The scaffold crystallinity did not change significantly with crosslinker type (GN versus TPP). The infrared spectra of chitosan (CS), CBT, and CBG scaffolds with $0.1 \mathrm{w} / \mathrm{v}$ crosslinker are shown in Figure $2 \mathrm{~b}$. The absorptions with peak position at $1656 \mathrm{~cm}^{-1}$ was assigned to $\mathrm{C}=\mathrm{O}$ stretching of the amide groups (amide I) of chitosan [32]. The absorption in the $1530-1580 \mathrm{~cm}^{-1}$ region was assigned to $\mathrm{N}-\mathrm{H}$ bending and $\mathrm{C}-\mathrm{N}$ stretching vibrations of the amide groups (amide II) of chitosan [32]. The strong absorption overlapping other peaks with position at $1200 \mathrm{~cm}^{-1}$ was assigned to P-O stretching of TPP in CBT [18]. A noticeable shift in the amide N-H absorption of chitosan in the $1550-1570 \mathrm{~cm}^{-1}$ region was attributed to the interaction between chitosan and genipin in $\mathrm{CBG}$.

Surface hydrophilicity affects protein adsorption and cell spreading on the surface of the scaffold [4]. The contact angle of water on GN and TPP crosslinked scaffolds as a function of crosslinker concentration is compared with those of CS and CS/ $\beta$-TCP in Table 1 . The surface hydrophilicity of the scaffolds decreased with TPP or GN crosslinking as the water contact angle increased (Table 1). As the TPP concentration increased from $0.01 \mathrm{w} / \mathrm{v}$ to $0.05,0.1,0.15$ and 0.2 w/v, the water contact angle on CBT scaffolds increased from $52.5 \pm 0.5^{\circ}$ to $53.4 \pm 0.9,54 \pm 1.1$, $54.6 \pm 0.7$ and $54.9 \pm 0.6^{\circ}$, respectively; the contact angle on CBG scaffolds increased from $54.2 \pm 0.4^{\circ}$ to $54.7 \pm 0.6,55.1 \pm 1,55.6 \pm 0.7$ and $56.1 \pm 0.5^{\circ}$ (Table 1 ). Overall, the water contact angle on CBG scaffolds was higher (statistically significant) than the CBT scaffolds which was related to the lower porosity of $\mathrm{CBG}$ scaffolds (lower fraction of water-filled pores on the surface).

\subsection{Compressive strength, swelling and degradation}


The compressive strength of CS/nano $\beta$-TCP scaffolds showed a bimodal trend with TPP/GN crosslinking, increasing slightly at lower crosslinker concentrations $(0.01-0.1 \mathrm{w} / \mathrm{v})$ but decreasing significantly at higher concentrations of 0.15 and $0.2 \mathrm{w} / \mathrm{v}$. As the TPP concentration increased from $0.01 \mathrm{w} / \mathrm{v}$ to $0.05,0.1,0.15$ and $0.2 \mathrm{w} / \mathrm{v}$, the compressive strength of the CBT scaffolds changed from 2.69 $\pm 0.17 \mathrm{MPa}$ to $2.71 \pm 0.11,2.72 \pm 0.19,2.59 \pm 0.19$ and $2.34 \pm 0.14 \mathrm{MPa}$, respectively, whereas the compressive strength of the CBG scaffolds changed from $2.69 \pm 0.11$ MPa to $2.74 \pm 0.13,2.78 \pm 0.14,2.65 \pm 0.17$ and $2.49 \pm 0.19 \mathrm{MPa}$ (Figure $3 \mathrm{a}$ and Table 2). The initial increase in compressive strength with crosslinker concentration can be attributed to the lower porosity of CBT and CBG scaffolds compared to the uncrosslinked CS/ $\beta$-TCP scaffold (Table 2). The reduction in compressive strength at higher crosslinker concentrations was most likely due to the increase in average pore size (Table 2). Overall, there was not a statistically significant difference between the compressive strength of CBT and CBG scaffolds. Figure 3b shows the swelling of the scaffolds in aqueous solution with incubation time. All scaffolds reached equilibrium swelling in $<6 \mathrm{~h}$. Overall the swelling of CBT scaffolds was statistically higher than the CBG scaffolds but both CBG and CBT scaffolds had lower swelling compared to $\mathrm{CS}$ and $\mathrm{CS} / \beta$-TCP scaffolds (Table 2). Further, percent swelling decreased with increasing TPP or GN concentration. The decrease in swelling of TPP and GN crosslinked scaffolds was attributed to their lower porosity (Table 1).

The remaining mass of the scaffolds with incubation time is shown in Figure 3c. The mass loss after 30 days of incubation is given in Table 2. The mass loss of CBT scaffolds was higher than the CBG scaffolds and showed a bimodal trend with the crosslinker concentration. The scaffold mass loss after 30 days changed from $14 \%$ to $11,8,10$, and $12 \%$ as GN concentration increased from zero to $0.01,0.05,0.1,0.15$ and $0.2 \mathrm{w} / \mathrm{v}$, respectively. At a given crosslinker 
concentration, the difference in mass loss between CBT and CBG scaffolds was not statistically significant [20].

\subsection{Cell viability and osteogenic differentiation}

Since CBT and CBG scaffolds with $0.1 \mathrm{w} / \mathrm{v}$ crosslinker concentration had higher compressive strength and mass loss compared to other concentrations (Table 2), CBT0.1 and CBG0.1 scaffolds were selected for osteogenic differentiation experiments with hMSCs. The SEM images in Figure 4(a-d) and Figure 4(e-h) show the cell-seeded CBG0.1 and CBT0.1 scaffolds, respectively, after 1 (a and e), 3 (b and f), 7 ( $\mathrm{c}$ and $\mathrm{g}$ ) and $14(\mathrm{~d}$ and $\mathrm{h}$ ) days of incubation in osteogenic medium. Images indicate that ECM deposition by the seeded cells increased with incubation time, especially after 7 and 14 days of incubation. Metabolic activity of hMSCs seeded on CBG and CBT scaffolds is compared with that of chitosan scaffold in Figure 5A after 1,3 , and 5 days of incubation in osteogenic medium . Metabolic activity of hMSCs in all groups increased with time. Metabolic activity of the hMSCs seeded in CBG and CBT scaffolds was significantly higher than the hMSCs seeded in the CS scaffold for all time points. The metabolic activity of the hMSCs seeded in CBG scaffolds was slightly higher (statistically significant) than the hMSCs seeded in CBT. Wang et al. evaluated toxicity of silk fibroin scaffolds crosslinked with genipin, glutaraldehyde, or polyethylene glycol diglycidyl ether with L929 mouse fibroblast cells. He reported higher growth and lower apoptosis of L929 cells seeded on GN crosslinked scaffolds compared to the other crosslinkers [33]. Lima et al. reported that crosslinking of chitosan membranes with TPP had no effect on cell viability [34]. Therefore, the increase in metabolic activity of hMSCs seeded in CBG scaffolds is indicative of the low toxicity of GN and TPP crosslinkers [21,35]. Fluorescent images in Figure 5B show that the seeded cells grew significantly from day 3 (image a for GN and c for TPP) to day 14 (image b 
for GN and d for TPP). The cell morphology was spherical initially but cell spreading increased with incubation time and the density of dead cells (stained red) in the images of Figure 5B decreased with incubation time. The organization of hMSCs seeded in CBG scaffolds was imaged by confocal microscopy and the results are shown in Figure 6 after 7 (image a) and 14 days (b) of incubation in osteogenic medium. The images show the spread morphology of the cells and the increase in cellularity with incubation time. The z-stacked 3D images show cell penetration in a $50 \mu \mathrm{m}$ section of the scaffold (images c and d in Figure 6). Similar patterns of cell penetration and cell distribution were observed previously by fibroblasts seeded in alginate/ $\beta$-TCP composite scaffolds [36].

The cell content, ALP activity, and calcium content of hMSCs seeded in CBG0.1 and CBT0.1 scaffolds are shown in Figure 7a through 7c, respectively, as a function of incubation time. For all time points, the cell content of CBG scaffolds was slightly higher than CBT and the difference was statistically significant with $452 \pm 22 \mathrm{ng} / \mathrm{mL}$ and $376 \pm 13 \mathrm{ng} / \mathrm{mL}$ for CBG and CBT, respectively, after 21 days of incubation (Figure 7a). ALP activity of hMSCs seeded in CBG and CBT scaffolds was bimodal with respect to incubation time which increased from day zero to 7 and 14 and then decreased from day 14 to 21 (Figure $7 b$ ), consistent with the pattern of ALP production by MSCs undergoing osteogenesis [37]. For both CBG and CBT scaffolds, the peak ALP activity of the hMSCs was observed on day $14(6.09 \pm 0.06 \mathrm{IU} / \mu \mathrm{g}$ for $\mathrm{CBG}$ and $5.5 \pm 0.07 \mathrm{IU} / \mu \mathrm{g}$ for CBT). ALP activity of hMSCs in CBG scaffolds on days 7 and 14 was slightly higher than the cells in CBT scaffolds but the difference was not significant. Calcium content as a measure of the extent of mineralization of the seeded hMSCs increased steadily with incubation time (Figure 7c). Calcium content of the hMSCs seeded in CBG scaffolds was slightly higher than the cells in CBT scaffolds in days 7 and 14 but the difference was not 
statistically significant. However, the extent of mineralization of hMSC-seeded CBG scaffolds after 21 days of incubation in osteogenic medium was significantly higher than the CBT scaffolds. After 21 days of incubation, calcium content of hMSC-seeded CBG scaffolds was $2050 \pm 130 \mathrm{mg} / \mathrm{mg}$ DNA whereas that of CBT scaffolds was $1860 \pm 100 \mathrm{mg} / \mathrm{mg}$ DNA.

In summary, there was a statistically significant difference between the average pore size, porosity, contact angle, and percent swelling of CBT and CBG scaffolds. The average pore size of CBG scaffolds was higher than CBT, the porosity of $\mathrm{CBG}$ was lower than CBT, the water contact angle of CBG was higher than CBT, and the percent swelling of CBG was lower than CBT. Taken together, the results demonstrate that freeze-gelled chitosan/nano $\beta$-TCP scaffolds crosslinked with genipin had higher average pore size, lower porosity, lower water contact angle, and lower swelling compared to the un-crosslinked chitosan/ $\beta$-TCP. Further, CBG scaffolds had slightly higher extent of mineralization after 21 days incubation in osteogenic medium compared to CBT.

\section{Conclusions}

In this work, the method of freeze-gelation was used to synthesize porous chitosan/nano $\beta$-TCP scaffolds crosslinked with GN or TPP. The synthesized scaffolds were characterized with respect to porosity, pore size, percent swelling, wettability, compressive strength, and mass loss. The scaffolds were seeded with human MSCs and evaluated with respect to osteogenic differentiation with incubation time. Experimental groups included chitosan/ $\beta$-TCP scaffold crosslinked with TPP (CBT) and chitosan/ $\beta$-TCP scaffold crosslinked with GN (CBG). All scaffolds displayed interconnected honeycomb-like microstructures with porosities $>65 \%$. The scaffold pore size increased with GN or TPP crosslinking whereas the scaffold porosity decreased. Further, pore size of the scaffolds increased and porosity decreased with crosslinker 
concentration for both TPP and GN. The water contact angle on the scaffolds increased with increasing TPP or GN concentration but scaffold degradation did not change significantly with crosslinker type. Compressive strength of the chitosan scaffolds was bimodal with TPP/GN crosslinker concentration with an initial increase at lower concentration $(0.01-0.1 \mathrm{w} / \mathrm{v})$ followed by a decrease at higher concentrations $(0.15$ and $0.2 \mathrm{w} / \mathrm{v})$. This bimodal trend was attributed to the changes in porosity and pore size of the scaffolds with crosslinker concentration. Metabolic activity of hMSCs seeded in CBG scaffolds was significantly higher than CBT scaffolds. Furthermore, CBG scaffolds had slightly higher extent of mineralization after 21 days incubation in osteogenic medium compared to CBT scaffolds.

\section{Acknowledgements}

N. Siddiqui and K. Pramanik would like to thank the Department of Biotechnology, New Delhi, Government of India, for providing research facility under the program support on Tissue Engineering Research and National Institute of Technology, Rourkela for providing fellowship to one of the authors (Siddiqui) and other infrastructural support to carry out this work. This work was also supported by research grants to E. Jabbari from the National Science Foundation under grant numbers IIP-1357109 and CBET-1403545, and the National Institutes of Health under Grant number AR063745. The authors thank Dr. Tahereh Karimi and Dr. Seyedsina Moeinzadeh for assistance with cell cultivation and data analysis. 


\section{References}

[1] L. Bi, Z. Cao, Y. Hu, Y. Song, L. Yu, B. Yang, J. Mu, Z. Huang, Y. Han, Effects of different crosslinking conditions on the properties of genipin-cross-linked chitosan/collagen scaffolds for cartilage tissue engineering, J. Mater. Sc. Mater. Med. 22 (2011) 51-62.

[2] S.H. Cartmell, B.D. Porter, A.J. García, R.E. Guldberg, Effects of medium perfusion rate on cellseeded three-dimensional bone constructs in vitro, Tissue Eng. 9 (2003) 1197-1203.

[3] K. Cheng, W.S. Kisaalita, Exploring cellular adhesion and differentiation in a micro-/nano-hybrid polymer scaffold, Biotechnol. Prog. 26 (2010) 838-846.

[4] A. Di Martino, M. Sittinger, M.V. Risbud, Chitosan: a versatile biopolymer for orthopaedic tissueengineering, Biomaterials 26 (2005) 5983-5990.

[5] C.G. Havens, A. Ho, N. Yoshioka, S.F. Dowdy, Regulation of late G1/S phase transition and apccdh1 by reactive oxygen species, Molecul. Cell. Biol. 26 (2006) 4701-4711.

[6] M.-H. Ho, P.-Y. Kuo, H.-J. Hsieh, T.-Y. Hsien, L.-T. Hou, J.-Y. Lai, D.-M. Wang, Preparation of porous scaffolds by using freeze-extraction and freeze-gelation methods, Biomaterials 25 (2004) $129-138$.

[7] C.-Y. Hsieh, S.-P. Tsai, M.-H. Ho, D.-M. Wang, C.-E. Liu, C.-H. Hsieh, H.-C. Tseng, H.-J. Hsieh, Analysis of freeze-gelation and cross-linking processes for preparing porous chitosan scaffolds, Carbohyd. Polym. 67 (2007) 124-132.

[8] S.-h. Hsu, S.W. Whu, C.-L. Tsai, Y.-H. Wu, H.-W. Chen, K.-H. Hsieh, Chitosan as scaffold materials: Effects of molecular weight and degree of deacetylation, J. Polym. Res. 11 (2004) 141147.

[9] D.W. Hutmacher, Scaffolds in tissue engineering bone and cartilage, Biomaterials 21 (2000) 25292543.

[10] C. Ji, N. Annabi, A. Khademhosseini, F. Dehghani, Fabrication of porous chitosan scaffolds for soft tissue engineering using dense gas $\mathrm{CO}_{2}$, Acta Biomaterialia 7 (2011) 1653-1664.

[11] Y. Zhang, M. Ni, M. Zhang, B. Ratner, Calcium phosphate-chitosan composite scaffolds for bone tissue engineering, Tissue Eng. 9 (2003) 337-345.

[12] S. Rodrigues, A.M. Costa, A. Grenha, Chitosan/carrageenan nanoparticles: effect of cross-linking with tripolyphosphate and charge ratios, Carbohyd. Polym. 89 (2012) 282-289.

[13] W. Thein-Han, R. Misra, Biomimetic chitosan-nanohydroxyapatite composite scaffolds for bone tissue engineering, Acta Biomaterialia 5 (2009) 1182-1197.

[14] W. Xu, J. Ma, E. Jabbari, Material properties and osteogenic differentiation of marrow stromal cells on fiber-reinforced laminated hydrogel nanocomposites, Acta Biomaterialia 6 (2010) 1992-2002.

[15] N. Siddiqui, K. Pramanik, Effects of micro and nano $\beta$-TCP fillers in freeze-gelled chitosan scaffolds for bone tissue engineering, J. Appl. Polym. Sci. 131 (2014) DOI: 10.1002/APP.41025.

[16] N. Siddiqui, K. Pramanik, Development of fibrin conjugated chitosan/nano $\beta$-TCP composite scaffolds with improved cell supportive property for bone tissue regeneration, J. Appl. Polym. Sci. (2015). DOI: 10.1002/app.41534.

[17] L.L. Huang-Lee, D.T. Cheung, M.E. Nimni, Biochemical changes and cytotoxicity associated with the degradation of polymeric glutaraldehyde derived crosslinks, J. Biomed. Mater. Res. 24 (1990) $1185-1201$.

[18] F. Pati, B. Adhikari, S. Dhara, Collagen intermingled chitosan-tripolyphosphate nano/micro fibrous scaffolds for tissue-engineering application, J. Biomater. Sci. Polym. Ed. 23 (2012) 1923-1938.

[19] F. Pati, H. Kalita, B. Adhikari, S. Dhara, Osteoblastic cellular responses on ionically crosslinked chitosan-tripolyphosphate fibrous 3-d mesh scaffolds, J. Biomed. Mater. Res. Part A 101 (2013) 2526-2537. 
[20] J. Qiu, J. Li, G. Wang, L. Zheng, N. Ren, H. Liu, W. Tang, H. Jiang, Y. Wang, In vitro investigation on the biodegradability and biocompatibility of genipin cross-linked porcine acellular dermal matrix with intrinsic fluorescence, ACS Appl. Mater. Interf. 5 (2012) 344-350.

[21] M. Sarem, F. Moztarzadeh, M. Mozafari, How can genipin assist gelatin/carbohydrate chitosan scaffolds to act as replacements of load-bearing soft tissues?, Carbohyd. Polym. 93 (2013) 635-643.

[22] A. Subramanian, H.Y. Lin, Crosslinked chitosan: Its physical properties and the effects of matrix stiffness on chondrocyte cell morphology and proliferation, J. Biomed. Mater. Res. Part A 75 (2005) 742-753.

[23] F. Causa, P. Netti, L. Ambrosio, G. Ciapetti, N. Baldini, S. Pagani, D. Martini, A. Giunti, Poly- $\epsilon$-caprolactone/hydroxyapatite composites for bone regeneration: In vitro characterization and human osteoblast response, J. Biomed. Mater. Res. Part A 76 (2006) 151-162.

[24] F. Pati, B. Adhikari, S. Dhara, Development of chitosan-tripolyphosphate non-woven fibrous scaffolds for tissue engineering application, J. Mater. Sci. Mater. Med. 23 (2012) 1085-1096.

[25] H.M. Kim, F. Miyaji, T. Kokubo, T. Nakamura, Preparation of bioactive ti and its alloys via simple chemical surface treatment, J. Biomed. Mater. Res. 32 (1996) 409-417.

[26] S.M. Vickers, L.S. Squitieri, M. Spector, Effects of cross-linking type II collagen-GAG scaffolds on chondrogenesis in vitro: dynamic pore reduction promotes cartilage formation, Tissue Eng. 12 (2006) 1345-1355.

[27] H. Shearer, M.J. Ellis, S.P. Perera, J.B. Chaudhuri, Effects of common sterilization methods on the structure and properties of poly (d, 1 lactic-co-glycolic acid) scaffolds, Tissue Eng. 12 (2006) 27172727.

[28] E. Jabbari, X. He, M.T. Valarmathi, A.S. Sarvestani, W. Xu, Material properties and bone marrow stromal cells response to in situ crosslinkable rgd-functionlized lactide-co-glycolide scaffolds, $J$. Biomed. Mater. Res. Part A 89 (2009) 124-137.

[29] S. Moeinzadeh, D. Barati, X. He, E. Jabbari, Gelation characteristics and osteogenic differentiation of stromal cells in inert hydrolytically degradable micellar polyethylene glycol hydrogels, Biomacromolecules 13 (2012) 2073-2086.

[30] D. Barati, S. Moeinzadeh, O. Karaman, E. Jabbari, Time dependence of material properties of polyethylene glycol hydrogels chain extended with short hydroxy acid segments, Polymer 55 (2014) 3894-3904.

[31] S.S.A. Abidi, Q. Murtaza, Synthesis and characterization of nano-hydroxyapatite powder using wet chemical precipitation reaction, J. Mater. Sci. Technol. 30 (2014) 307-310.

[32] K. Van de Velde, P. Kiekens, Structure analysis and degree of substitution of chitin, chitosan and dibutyrylchitin by ft-ir spectroscopy and solid state c-13 nmr, Carbohyd. Polym. 58 (2004) 409-416.

[33] L. Wang, Y. Wang, J. Qu, Y. Hu, R. You, M. Li, The cytocompatibility of genipin-crosslinked silk fibroin films, J. Biomater. Nanobiotechnol. 4 (2013) 213-221.

[34] H.A. Lima, F.M.V. Lia, S. Swarnakar Ramdayal, Preparation and characterization of chitosaninsulin-tripolyphosphate membrane for controlled drug release: Effect of cross linking agent, $J$. Biomater. Nanobiotechnol. 5 (2014) 211-219.

[35] L.P. Yan, Y.J. Wang, L. Ren, G. Wu, S.G. Caridade, J.B. Fan, L.Y. Wang, P.H. Ji, J.M. Oliveira, J.T. Oliveira, Genipin-cross-linked collagen/chitosan biomimetic scaffolds for articular cartilage tissue engineering applications, J. Biomed. Mater. Res. Part A 95 (2010) 465-475.

[36] G. Diogo, V. Gaspar, I. Serra, R. Fradique, I. Correia, Manufacture of $\beta$-tcp/alginate scaffolds through a fab@ home model for application in bone tissue engineering, Biofabrication 6 (2014) 025001.

[37] S. Panzavolta, M. Fini, A. Nicoletti, B. Bracci, K. Rubini, R. Giardino, A. Bigi, Porous composite scaffolds based on gelatin and partially hydrolyzed $\alpha$-tricalcium phosphate, Acta biomaterialia 5 (2009) 636-643. 


\section{Figure Captions}

Figure 1. SEM image of CS/nano $\beta$-TCP/GN scaffolds crosslinked with 0.01 (a), 0.1 (b), and 0.2 (c) w/v GN. SEM image of CS/nano $\beta$-TCP/TPP scaffolds crosslinked with 0.01 (d), 0.1 (e), and 0.2 (f) w/v TPP.

Figure 2. XRD (a) and infrared (b) spectra of chitosan (CS, black), GN-crosslinked chitosan/ $\beta$ TCP (CBG, purple), and TPP-crosslinked chitosan/ $\beta$-TCP (CBT, green) scaffolds with $0.1 \mathrm{w} / \mathrm{v}$ concentration of GN or TPP crosslinker.

Figure 3. (a) Compressive strength of chitosan/nano $\beta$-TCP scaffolds crosslinked with GB (CBG, purple) and TPP (CBT, green)) as a function of crosslinker concentration; (b) water uptake of CBG (purple) and CBT (green) scaffolds with $0.05 \mathrm{w} / \mathrm{v}$ crosslinker concentration (CBG0.05 and CBT0.05) with incubation time; (c) mass loss of CBG (purple) and CBT (green) scaffolds with $0.1 \mathrm{w} / \mathrm{v}$ crosslinker concentration with incubation time.

Figure 4. SEM images of GN (a-d) and TPP (e-h) crosslinked chitosan/nano $\beta$-TCP scaffolds seeded with hMSCs and incubated in osteogenic medium for 1 (a,e), 3 (b,f), 7 (c,g), and 14 (d,h) days. ECM secretion by the cultured hMSCs on the scaffold surface is evident as represented by arrows.

Figure 5. (A) Metabolic activity of hMSCs seeded in chitosan scaffold (CS, dark blue), GNcrosslinked chitosan/nano $\beta$-TCP (CBG0.1, purple), and TPP-crosslinked chitosan/nano $\beta$-TCP (CBT0.1, green) scaffolds with $0.1 \mathrm{w} / \mathrm{v}$ crosslinker concentration. Each point represents the mean $\pm \mathrm{SD}(\mathrm{n}=3)$. A star shows a statistically significant $(\mathrm{p}<0.05)$ difference between the CBG and CBT groups at the same time point. Live/dead fluorescent images of the adhered hMSCs seeded in the scaffold after 3 days (a for GN and c for TPP) and 14 days (b for GN and d for TPP). The scale bar in a-d is $200 \mu \mathrm{m}$.

Figure 6. (a) Confocal laser scanning microscopic images of hMSCs cultured on CBG scaffolds after 7 (a) and 14 (b) days incubation in osteogenic medium. Images (c) and (d) are $50 \mu \mathrm{m} \mathrm{z-}$ stacked confocal images of the hMSC-seeded CBG scaffold after 14 days incubation in the $\mathrm{x}-\mathrm{y}$ plane and the thickness direction (z plane), respectively. The scale bar in a-d is $300 \mu \mathrm{m}$.

Figure 7. DNA content (a), ALP activity (b), and calcium content (c) of hMSCs seeded in CBG $(0.1 \mathrm{w} / \mathrm{v}$ GN, purple) and CBT (0.1w/v TPP, green) scaffolds with incubation time in osteogenic medium. "A star" indicates a statistically significant difference between the test time point and previous time point for a given group. "Two stars" indicates a statistically significant difference between the two groups for a given time point. 
Table 1. Pore size, porosity, and contact angle of tripolyphosphate (CBT) and genipin (CBG) crosslinked chitosan/nano $\beta$-TCP scaffolds. CS and CS/ $\beta$-TCP scaffolds are chitosan and chitosan/nano $\beta$-TCP scaffolds, respectively, without TPP or GN crosslinking. The numbers next to CBT and CBG represent the concentration of crosslinker (w/v).

\begin{tabular}{|c|c|c|c|}
\hline Sample & Pore size $(\mu \mathrm{m})$ & Porosity \% & Contact angle $\left({ }^{\circ}\right)$ \\
\hline $\mathrm{CS}$ & $171 \pm 8$ & $86 \pm 3$ & $61.0 \pm 1.0$ \\
\hline $\mathrm{CS} / \beta-\mathrm{TCP}$ & $165 \pm 9$ & $83 \pm 5$ & $63.0 \pm 0.9$ \\
\hline CBT 0.01 & $168 \pm 4$ & $78 \pm 3$ & $52.5 \pm 0.5$ \\
\hline CBT 0.05 & $171 \pm 9$ & $76 \pm 1$ & $53.4 \pm 0.9$ \\
\hline CBT 0.10 & $175 \pm 8$ & $75 \pm 2$ & $54.0 \pm 1.1$ \\
\hline CBT 0.15 & $179 \pm 6$ & $73 \pm 2$ & $54.6 \pm 0.7$ \\
\hline CBT 0.20 & $186 \pm 5$ & $71 \pm 2$ & $54.9 \pm 0.6$ \\
\hline CBG 0.01 & $171 \pm 8$ & $76 \pm 3$ & $54.2 \pm 0.4$ \\
\hline CBG 0.05 & $179 \pm 7$ & $74 \pm 2$ & $54.7 \pm 0.6$ \\
\hline CBG 0.10 & $183 \pm 6$ & $73 \pm 2$ & $55.1 \pm 1.0$ \\
\hline CBG 0.15 & $191 \pm 8$ & $70 \pm 2$ & $55.6 \pm 0.7$ \\
\hline CBG 0.20 & $199 \pm 9$ & $68 \pm 2$ & $56.1 \pm 0.5$ \\
\hline
\end{tabular}


Table 2. Percent swelling, compressive strength, and mass loss of tripolyphosphate (CBT) and genipin (CBG) crosslinked chitosan/nano $\beta$-TCP scaffolds. CS and CS/ $\beta$-TCP scaffolds are chitosan and chitosan/nano $\beta$-TCP scaffolds, respectively, without TPP or GN crosslinking. The numbers next to $\mathrm{CBT}$ and $\mathrm{CBG}$ represent the concentration of crosslinker (w/v).

\begin{tabular}{|l|l|l|l|}
\hline Sample & \% Swelling & $\begin{array}{l}\text { Compressive } \\
\text { strength }(\mathbf{M P a})\end{array}$ & \% Mass loss \\
\hline CS & $325 \pm 16$ & $0.18 \pm 0.05$ & 18 \\
\hline CS/ $\beta$-TCP & $302 \pm 12$ & $2.67 \pm 0.13$ & 15 \\
\hline CBT 0.01 & $258 \pm 11$ & $2.69 \pm 0.17$ & 16 \\
\hline CBT 0.05 & $246 \pm 7$ & $2.71 \pm 0.11$ & 15 \\
\hline CBT 0.10 & $240 \pm 10$ & $2.72 \pm 0.11$ & 13 \\
\hline CBT 0.15 & $231 \pm 8$ & $2.59 \pm 0.19$ & 15 \\
\hline CBT 0.20 & $222 \pm 9$ & $2.34 \pm 0.15$ & 16 \\
\hline CBG 0.01 & $231 \pm 6$ & $2.69 \pm 0.11$ & 14 \\
\hline CBG 0.05 & $225 \pm 7$ & $2.74 \pm 0.13$ & 11 \\
\hline CBG 0.10 & $214 \pm 7$ & $2.78 \pm 0.14$ & 8 \\
\hline CBG 0.15 & $201 \pm 6$ & $2.65 \pm 0.17$ & 10 \\
\hline CBG 0.20 & $189 \pm 7$ & $2.49 \pm 0.10$ & 12 \\
\hline & & & \\
\hline
\end{tabular}




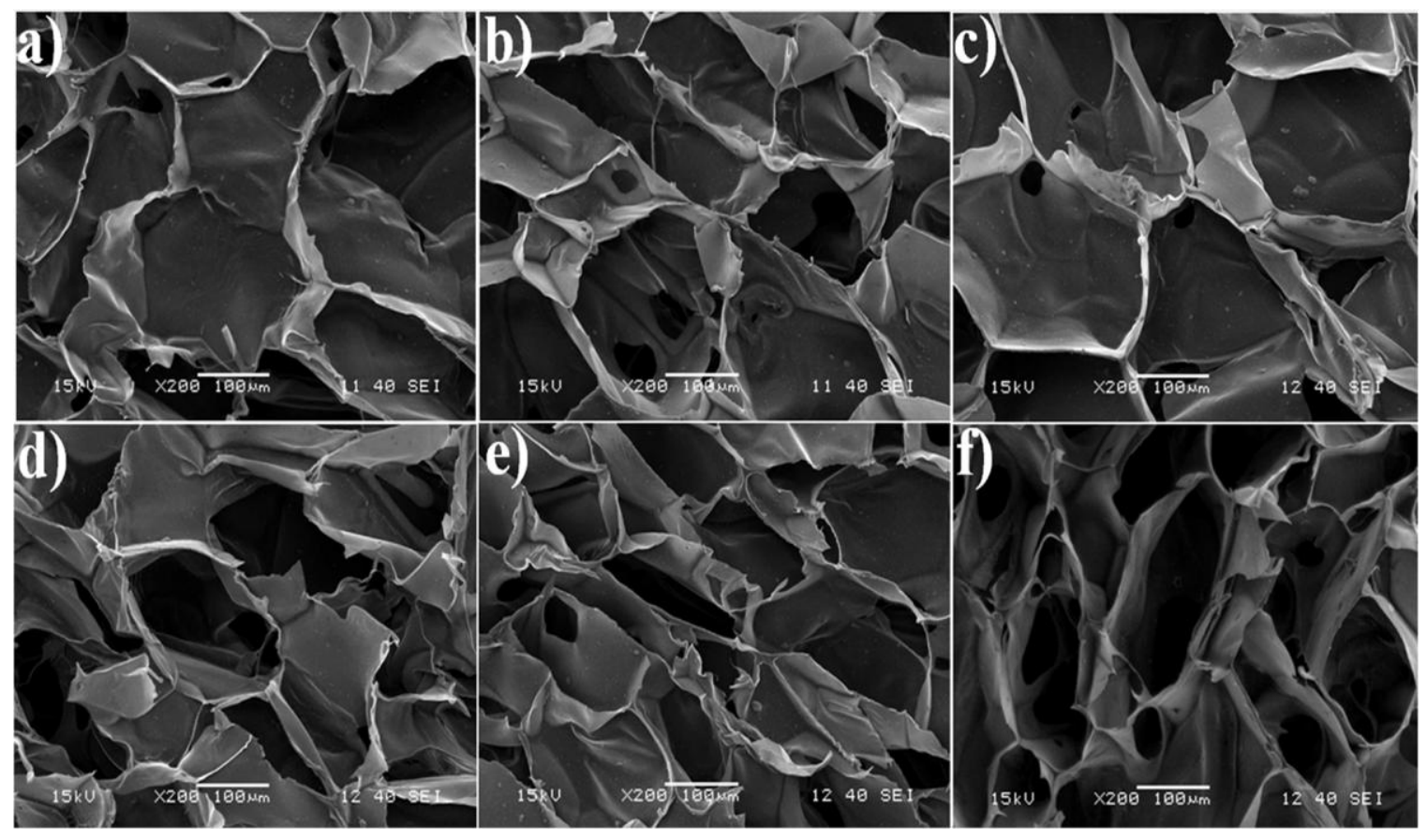

Figure 1 

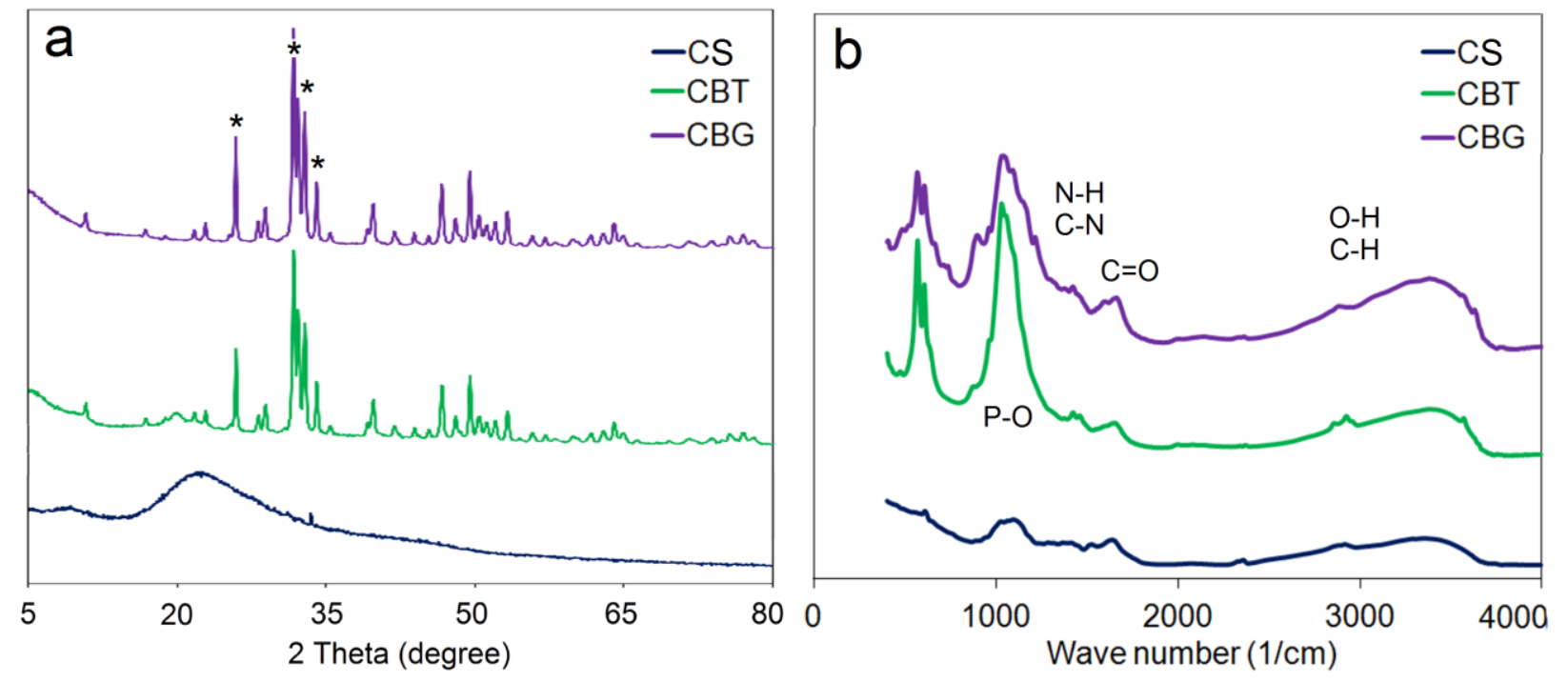

Figure 2 

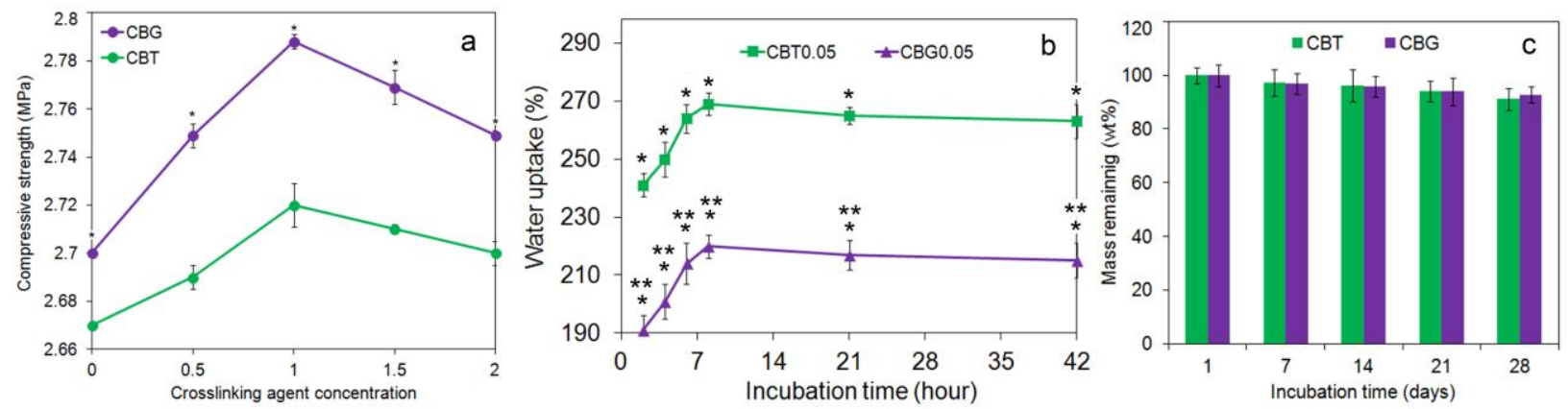

Figure 3 


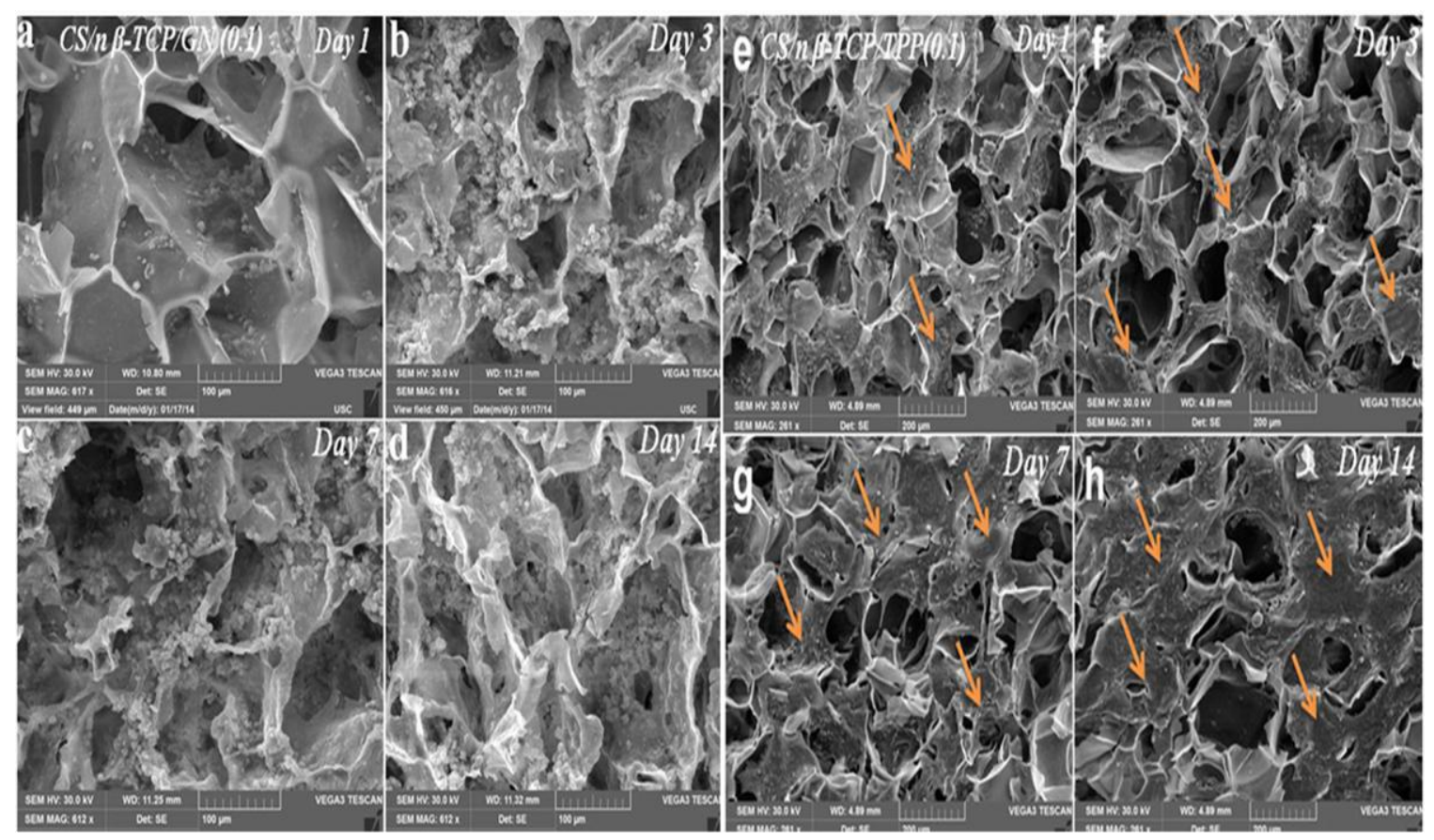

Figure 4 

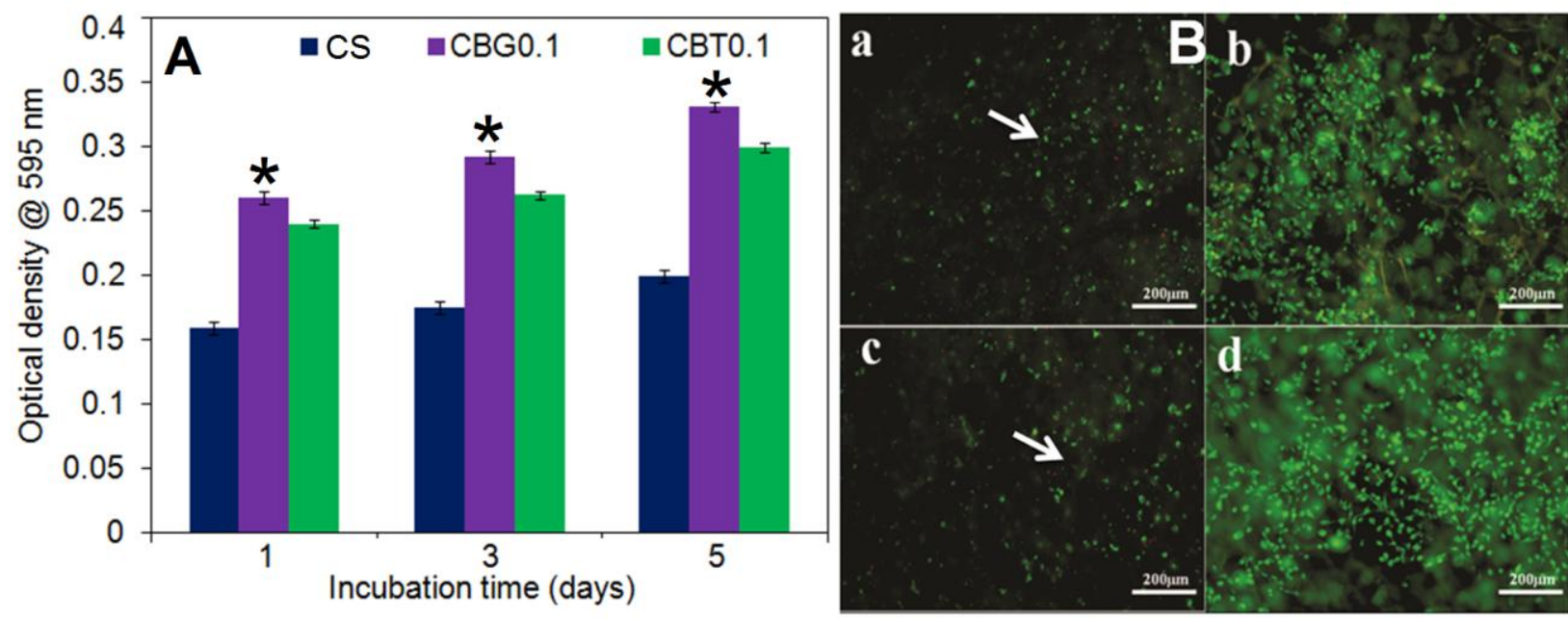

Figure 5 


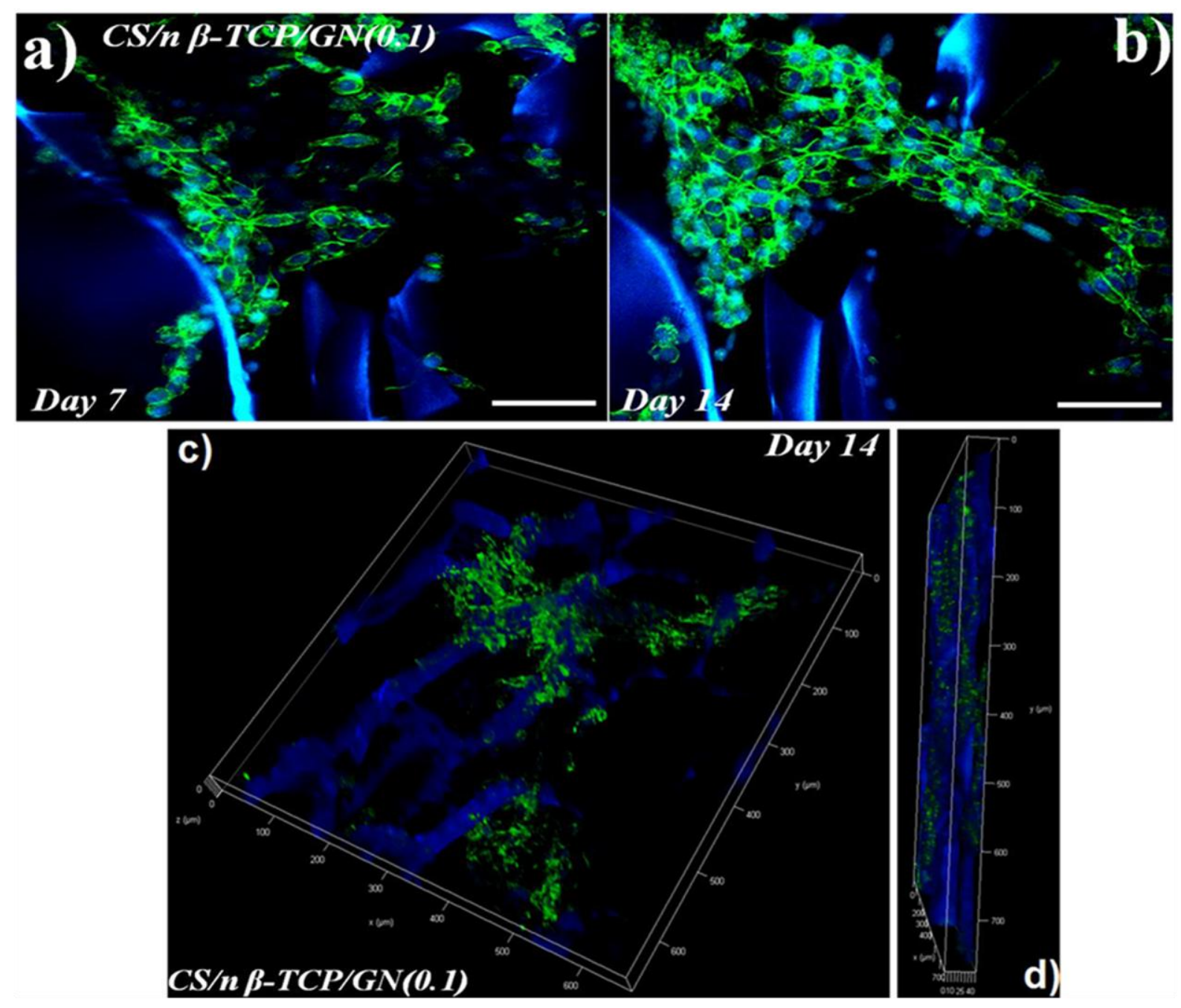

Figure 6 

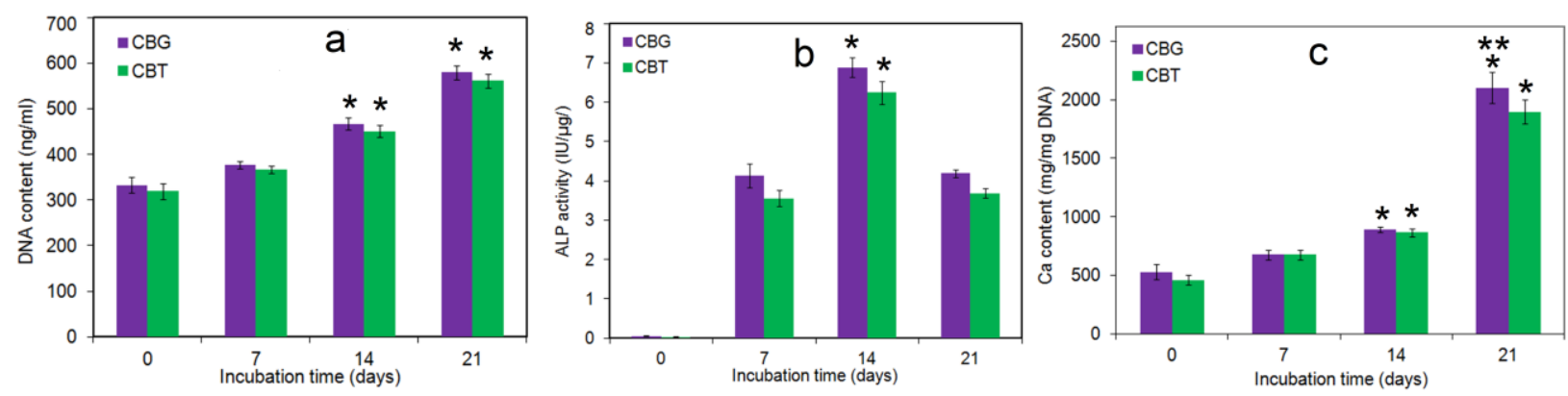

Figure 7 